Reprod. Nutr. Dévelop., 1988, 28 (1), 103-104.

\title{
Détermination du cobalt-EDTA dans les contenus duodénaux
}

\author{
J. BELLANGER
}

Laboratoire des Maladies Nutritionnelles,

I.N.R.A., Theix, 63122 Ceyrat, France.

Summary. Cobalt lithium ethylenediamine-tetraacetate (CoLiEDTA) and ytterbium chloride were used for estimating duodenal digesta flow in sheep. A method is described to measure cobalt in duodenal digesta by atomic absorption spectrometry.

Pour mesurer les flux duodénaux chez le mouton nous avons utilisé le principe de la méthode du double marquage (Faichney, 1980). L'ytterbium a été choisi comme marqueur de la phase solide et le complexe cobalt lithium-acide éthylènediamine-tétraacétique (CoLiEDTA) comme marqueur de la phase liquide. Le cobalt est déterminé par spectrométrie d'absorption atomique.

Le complexe CoLiEDTA de l'échantillon ne permet pas le dosage direct du cobalt par dilution. La méthode des ajouts dosés est longue et elle peut conduire à des résultats erronés (Montiel et Welte, 1986 ; Welz, 1986). C'est pourquoi nous avons développé une méthode de destruction de la matière organique par voie sèche. L'étalonnage s'effectue dans une solution duodénale témoin $(0 \mathrm{Co})$ après minéralisation.

Matériel et méthodes. Les essais de mise au point de la méthode sont conduits avec des solutions, de CoLiEDTA ou CoNa ${ }_{2}$ EDTA $(0,5 \mathrm{mg} \mathrm{Co} / \mathrm{ml})$ et d'ytterbium ( $1 \mathrm{mg} / \mathrm{ml})$ qui sont perfusées en continu $(200 \mathrm{ml} / 24 \mathrm{~h})$ dans le rumen de moutons fistulés. Les échantillons duodénaux sont centrifugés à $50000 \mathrm{~g}$ pendant $30 \mathrm{~min}$. Les phases liquide et solide sont ensuite séparées.

La prise d'essai de $5 \mathrm{ml}$ est évaporée à sec, dans un tube à essai, à l'étuve à $80^{\circ} \mathrm{C}$ puis minéralisée au four à $500^{\circ} \mathrm{C}$ pendant $10 \mathrm{~h}$ avec un palier à $350^{\circ} \mathrm{C}$ pendant $1 \mathrm{~h}$.

Les cendres sont reprises par $2 \mathrm{ml}$ d'acide chlorhydrique $6 \mathrm{M}$ et homogénéisées aux ultra-sons pendant $30 \mathrm{~min}$. La solution est chauffée à $100{ }^{\circ} \mathrm{C}$ pendant $30 \mathrm{~min}$ dans un bloc chauffant. La solution refroidie est ensuite diluée à $20 \mathrm{ml}$ avec de l'eau désionisée. Les dilutions éventuelles sont faites avec une solution chlorhydrique $0,6 \mathrm{M}$ du duodénum témoin. Le cobalt est déterminé par spectrométrie d'absorption atomique avec une flamme air-acétylène stœchiométrique à 240,7 nm. Un échantillon témoin, prélevé sur un mouton non 
perfusé en cobalt, nourri avec le même régime, est utilisé pour préparer les solutions étalons $(0,25 ; 0,5 ; 1 ; 1,5$ et $2 \mu \mathrm{g} \mathrm{Co} / \mathrm{ml}$. La prise d'essai $(25 \mathrm{ml})$ est évaporée dans une capsule en porcelaine et traitée de même manière que les échantillons ; le cobalt $\left(\mathrm{CoCl}_{2}\right)$ est ajouté à la reprise des cendres dans une fiole de $100 \mathrm{ml}$. Une solution de dilution (témoin sans ajout de cobalt) et un essai à blanc sont conduits de manière identique aux étalons.

Résultats et discussion. A partir d'une prise d'essai de $5 \mathrm{ml}$ repris dans $20 \mathrm{ml}$, la gamme étalon permet de déterminer de 1 à $8 \mu \mathrm{g} / \mathrm{ml}$ de cobalt dans la phase liquide. Par contre le cobalt alimentaire n'est pas détectable dans l'échantillon provenant du mouton témoin.

Les perturbations (matrice, acide, ytterbium) ont été étudiées en ajoutant des quantités croissantes de l'élément perturbateur et en mesurant l'effet sur l'absorption du cobalt (tabl. 1). L'effet de I'EDTA ne peut être mesuré en augmentant le pourcentage d'EDTA puisque dans les échantillons le rapport EDTA/Co reste constant et propre à chaque complexe ( $\mathrm{Li}$ ou $\mathrm{Na}$ ). Les sels CoLiEDTA et CoNa ${ }_{2}$ EDTA ont été synthétisés (Udén et al., 1980) puis dosés, soit après dilution, soit après destruction du complexe. Seule la deuxième méthode donne des résultats conformes aux quantités théoriques de cobalt dans les deux complexes.

TABL. 1. - Etude des perturbations du dosage du cobalt dans les contenus duodénaux.

\begin{tabular}{|c|c|c|c|}
\hline $\begin{array}{c}\text { Elément } \\
\text { perturbateur }\end{array}$ & Forme étudiée & $\begin{array}{l}\text { Variation } \\
\text { de I'absorption du Co }\end{array}$ & $\begin{array}{l}\text { Perturbation } \\
\text { supprimée par }\end{array}$ \\
\hline EDTA & $\begin{array}{l}\text { CoLiEDTA } \\
\text { CoNa } \\
\text { EDTA }\end{array}$ & Diminution & $\begin{array}{l}\text { Destruction } \\
\text { du complexe }\end{array}$ \\
\hline Matrice & $\begin{array}{l}\text { Jus de duodénum } \\
\text { témoin }\end{array}$ & Diminution & Matrice $\leqslant 25 \%$ \\
\hline Acide & Ac. chlorhydrique & Diminution & Molarité $<1,5 \mathrm{M}$ \\
\hline Ytterbium & Chlorure & Pas d'effet & - \\
\hline
\end{tabular}

La répétabilité varie peu en fonction de la concentration : Jus duodénum : $\mathrm{C}=7,09 \mu \mathrm{g} / \mathrm{ml} \quad \mathrm{CV}=1,04 \%(\mathrm{n}=10)$; Jus duodénum : $\mathrm{C}=22,04 \mu \mathrm{g} / \mathrm{ml} \quad \mathrm{CV}=1,54 \%(\mathrm{n}=10)$; Sol. CoLiEDTA : $\mathrm{C}=1490 \mu \mathrm{g} / \mathrm{ml} \quad \mathrm{CV}=0,49 \%(\mathrm{n}=10)$.

La récupération $(R)$ après minéralisation d'ajouts de chlorure de cobalt varie en fonction de la matrice :

$\mathrm{CoCl}_{2}$ dans l'eau : $\mathrm{R}=99,6 \% \quad \mathrm{CV}=0,66 \%$;

$\mathrm{CoCl}_{2}$ duodénum témoin : $\mathrm{R}=98,7 \% \mathrm{CV}=1,5 \%$;

La méthode est fiable et répétable. Elle peut être étendue aux fèces : $\mathrm{R}=99,1 \%$, répétabilité : $1,0 \%$. 